\title{
Developing an Evidence-based Followup Schedule for Bone Sarcomas Based on Local Recurrence and Metastatic Progression
}

\author{
Cara Cipriano MD, Anthony M. Griffin MSc, Peter C. Ferguson MD, \\ Jay S. Wunder MD
}

Published online: 23 June 2016

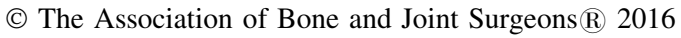

\begin{abstract}
Background The potential for local recurrence and pulmonary metastasis after treatment of primary bone sarcomas necessitates careful patient followup; however, minimal data exist regarding the incidence and timing of these events, and therefore an evidence-based surveillance protocol has not been developed.

Questions/purposes The purposes of this study were to (1) describe the frequency and timing of local recurrence by histologic grade over time; (2) describe the frequency and timing of metastasis by histologic grade and diagnosis
\end{abstract}

Each author certifies that he or she, or a member of his or her immediate family, has no funding or commercial associations (eg, consultancies, stock ownership, equity interest, patent/licensing arrangements, etc) that might pose a conflict of interest in connection with the submitted article.

All ICMJE Conflict of Interest Forms for authors and Clinical Orthopaedics and Related Research ${ }^{\circledR}$ editors and board members are on file with the publication and can be viewed on request.

Each author certifies that his or her institution approved the human protocol for this investigation, that all investigations were conducted in conformity with ethical principles of research, and that informed consent for participation in the study was obtained.

This work was performed at Mt Sinai Hospital, University of Toronto, Toronto, Ontario, Canada.

C. Cipriano ( $)$

Division of Orthopaedic Oncology, Department of Orthopaedic

Surgery, Washington University in St Louis, 660 S Euclid

Avenue, Campus Box 8233, St Louis, MO 63110, USA

e-mail: caracipriano@gmail.com

A. M. Griffin, P. C. Ferguson, J. S. Wunder

University Musculoskeletal Oncology Unit, Mount Sinai

Hospital, Toronto, ON, Canada

P. C. Ferguson, J. S. Wunder

Division of Orthopaedic Surgery, Department of Surgery,

University of Toronto, Toronto, ON, Canada over time; and (3) use these data to either justify current surveillance schedules and/or propose modifications that may improve the rate of new pulmonary metastatic events detected per examination.

Methods A retrospective review was performed of all patients who underwent resection of a primary, nonmetastatic bone sarcoma (excluding chordoma) at a single tertiary oncology center from 1989 to 2010 . Of the 680 patients identified, 15 were excluded for loss of followup in the first 2 years, leaving 665 eligible for study. Of these, 437 patients were alive with no evidence of disease at the conclusion of the study (mean followup, 136 months; range, 25-321 months). Cox regression analysis was performed to evaluate and control for patient age, tumor size, tumor location, and surgical margins. With patients stratified by sarcoma grade, Kaplan-Meier survival curves were constructed for the endpoints of local recurrence and metastasis, and log-rank tests were used to compare the rates of these events between grades and diagnoses. The number of new pulmonary metastatic events per patientyear was calculated for each sarcoma grade over the time intervals used in current surveillance protocols $(0-2,2-5$, $5-10$, and $>10$ years) to facilitate development of a surveillance schedule that would maximize events detected per imaging study performed. In addition, to determine the effect of disease type, subset analysis was performed for osteosarcoma (OSA) and chondrosarcoma because these were the only diagnoses with sufficient numbers to support individual statistical analysis.

Results With the numbers available for study, the overall local recurrence-free survival did not differ between sarcoma grades at any time points $(p=0.864)$. Metastasis-free survival curves differed between sarcoma grades $(\mathrm{p}<$ 0.001 ), and the pattern of Grade 2 OSA metastasis was more consistent with other Grade 3 sarcomas, so it was 
subsequently classified as high grade. No metastases of Grade 1 sarcomas occurred after 3 years, whereas Grade 2 and 3 sarcomas continued to metastasize until 10 years and rarely thereafter. According to the number of new pulmonary metastatic events per patient-year in each group, we propose that chest surveillance be performed according to the following schedule: annually only until 5 years for low-grade sarcomas; every 3 months for 2 years and annually from 2 to 10 years for intermediate-grade sarcomas; and every 3 months for 2 years, every 6 months from 2 to 5 years, and annually from 5 to 10 years for high-grade sarcomas.

Conclusions Pulmonary screening beyond 5 years may not be necessary for Grade 1 tumors but should be continued until 10 years for Grade 2 and 3 bone sarcomas. The surveillance frequency listed here, which is based on the number of new pulmonary metastatic events per patientyear in each grade, would increase the number of such events detected per examination performed.

Level of Evidence Level III, therapeutic study.

\section{Introduction}

After treatment for primary bone sarcoma, patients remain at risk for development of local and systemic disease recurrence, the latter occurring most commonly in the form of pulmonary metastasis. Although the importance of following patients with sarcoma is widely accepted, frequent surveillance is time-consuming for both patients and physicians, increases healthcare expenditures [11, 21, 22], and, with respect to the use of CT scans, exposes patients to ionizing radiation [4]. Furthermore, limited evidence exists to support the intervals, modalities, or duration of monitoring $[3,9,11]$.

The European Society for Medical Oncology (ESMO) recommends bone sarcoma followup with periodic clinical history, physical examination, and radiographic assessment of the tumor site as well as chest imaging (radiograph or
CT scan) for low- and high-grade sarcomas (Table 1) [8]. However, the authors also acknowledge that "strict rules cannot be provided in the absence of any formal validation," and the British Sarcoma Group echoes that "there is an urgent need for research" [12]. The National Cancer Institute recommends some form of chest imaging as followup but specifies neither modality nor timing. The only other guidelines available in the literature were issued by the National Comprehensive Cancer Network (NCCN), again based on scarce or no literature [18, 25]. In an effort to establish a basis of evidence to support these recommendations, two recent publications $[18,21]$ described the incidence and timing of local recurrence and distant metastasis in soft tissue sarcomas, confirming more frequent early disease progression in higher grade tumors [21]; to our knowledge, however, no such information on bone sarcomas has been published.

Thus, although some form of monitoring for local recurrence and pulmonary metastasis in bone sarcoma is universally practiced, it is based on minimal evidence and remains highly variable. The purpose of our study therefore was to address this issue by defining the risk of disease progression in terms of frequency, timing, and location and thereby providing guidance for development of an optimal surveillance schedule in these patients. We asked the following questions: (1) What is the frequency and timing of local recurrence by histologic grade over time? (2) What is the frequency and timing of metastasis by histologic grade and diagnosis over time? (3) Based on these findings, can we propose a surveillance schedule that maximizes events detected per examination?

\section{Patients and Methods}

The study cohort consisted of all patients who underwent surgical resection of a nonmetastatic primary bone sarcoma at a single tertiary oncology center from 1989 to 2010 . This retrospective study queried a longitudinally maintained

Table 1. Summary of ESMO and NCCN guidelines for bone sarcoma surveillance intervals as well as the protocol used at our institution over the course of this study

\begin{tabular}{lllll}
\hline Guideline & Grade & $0-2$ years & $3-4$ years & $5-10$ years \\
\hline ESMO & Low & 6 months & 12 months & 6 years \\
& High & $2-3$ months & $2-4$ months & 6 months \\
NCCN & Low & $6-12$ months & 12 months & 12 months \\
\multirow{3}{*}{ Study protocol } & High & $3-6$ months & & 12 months \\
& Low & 6 months & 6 months & 12 months \\
\hline
\end{tabular}

$\mathrm{ESMO}=$ European Society for Medical Oncology; NCCN = National Comprehensive Cancer Network. 
institutional sarcoma database for patient age and gender, sarcoma grade and histologic diagnosis (as determined by a board-certified pathologist), and location and timing of any disease progression. Chordoma was excluded as a result of its unique biology and patterns of treatment. Of the remaining 680 patients with primary, nonmetastatic bone sarcomas in our database, only 15 were excluded for less than 2 years of followup, leaving 665 available for the study. Histologic diagnoses included osteosarcoma (OSA; 281), chondrosarcoma (CSA; 245), Ewing's sarcoma (40), undifferentiated pleomorphic sarcoma (31), leiomyosarcoma (19), malignant giant cell tumor (GCT; seven), fibrosarcoma (13), adamantinoma (10), angiosarcoma (seven), and others (12). Three hundred twenty-three patients received chemotherapy and 35 were treated with radiotherapy (18 CSA, eight OSA, six Ewing's, and one each of fibrosarcoma, undifferentiated pleomorphic sarcoma, and other) [10]. There were 390 males (59\%) and 275 females (41\%) with 121 axial (18\%) and 544 appendicular $(82 \%)$ tumors.

Patients with low-grade sarcomas were followed every 6 months until 5 years, then annually, whereas those with intermediate- or high-grade sarcomas were followed every 3 months for the first 2 years, then every 6 months until 5 years, and annually thereafter. Each visit consisted of a clinical history, physical examination, and chest imaging. Patients with bone sarcoma who were treated with chemotherapy (intermediate- and high-grade tumors except CSAs) had CT chest imaging until 2 years and chest radiographs thereafter, whereas all other patients were followed with chest radiographs. Any suspicious findings on chest radiographs were then investigated with CT scans. Plain radiographs of the primary surgical site were typically obtained at each followup appointment, and crosssectional imaging was only requested based on suspicious findings on clinical examination or local radiographs. All imaging studies were reviewed by a board-certified radiologist in addition to the treating orthopaedic oncologist. At the conclusion of the study, 437 patients were alive with no evidence of disease (ANED), 20 were alive with evidence of disease, 168 had died of their disease, and 40 had died of unrelated causes. Of the ANED group, the mean followup was 136 months (range, 25-321 months); 24 patients had 2 to 5 years of followup, 167 had 5 to 10 years, and 263 had $>10$ years.

Kaplan-Meier curves were constructed for each sarcoma grade using local recurrence and lung metastasis as endpoints. Observed rates of disease progression were calculated, and the Breslow Day test was used to compare estimated survival between grades. Subgroup analyses were performed for development of metastatic disease in patients with OSA and CSA because these were the two most common diagnoses and were hypothesized to have distinct patterns of disease progression as a result of their differing pathophysiology and treatment protocols. Cox regression stratified by grade was also performed with patient age, tumor location (axial or appendicular), tumor size, and surgical margins (negative, microscopically positive, or grossly positive) analyzed as potential risk factors for lung metastasis. Analysis by diagnosis was not performed for local recurrence because of limited statistical power resulting from the lower incidence of these events. The overall Cox regression model for lung metastasis demonstrated only borderline significance for an alpha of 0.05 (chi square $=11.025, \mathrm{df}=5, \mathrm{p}=0.051$ ), and log minus $\log$ testing confirmed that the proportional hazard assumption was met for all variables. In addition, crude differences between sarcoma grades in the Kaplan-Meier analysis were examined in the risk-adjusted Cox regression model, confirming that these differences did not result from confounding.

The number of new pulmonary metastatic events per patient-year was calculated for each sarcoma grade over the time intervals used in current surveillance protocols ( 0 $2,2-5,5-10$, and $>10$ years). Incidence of metastases was then used to develop a potential surveillance protocol based on relative frequency of events to suggest a surveillance schedule that would maximize events detected per imaging study performed. Based on our findings, we proposed a followup protocol using the following criteria: chest imaging every 3 months for patients with 0.5 to 1.0 incidence of new pulmonary metastasis per patient-year, every 6 months for 0.1 to 0.5 incidence, every 12 months for 0.01 to 0.1 incidence, and no imaging for incidence $<0.01$. The resulting schedule was then compared with currently recommended protocols to identify any potential modifications that may improve the number of new pulmonary metastatic events detected per examination performed.

Statistical analysis was performed using SPSS software (Version 19.0; SPSS Inc, Chicago, IL, USA).

\section{Results}

With the numbers available for study, the observed local recurrence-free survival did not vary with increasing sarcoma grade at 2 years (Table 2). The log-rank test for overall local recurrence-free survival was not different among these three groups $(p=0.864)$ (Fig. 1). Late local recurrences were uncommon with only three cases in the fifth year, two in the sixth, one in the eighth, and one in the ninth year. Only one local recurrence occurred later than 10 years: an intermediate-grade CSA, which developed local recurrence 187 months after initial treatment and was followed shortly thereafter by development of lung 
Table 2. Observed local recurrence-free and metastasis-free survival at 2, 5, and 10 years compare rates between sarcoma grades and diagnoses with associated $95 \%$ confidence intervals

\begin{tabular}{|c|c|c|c|c|}
\hline Survival & Grade & 2-year \% (95\% CI) & 5 -year \% (95\% CI) & 10 -year \% $(95 \% \mathrm{CI})$ \\
\hline \multirow[t]{3}{*}{ Local recurrence-free survival, all diagnoses } & 1 & $97.1( \pm 2.7)$ & $91.8( \pm 4.7)$ & $89.02( \pm 5.3)$ \\
\hline & 2 & $94.3( \pm 2.9)$ & $90.7( \pm 3.9)$ & $89.1( \pm 4.3)$ \\
\hline & 3 & $90.5( \pm 3.5)$ & $90.0( \pm 3.7)$ & $88.5( \pm 4.1)$ \\
\hline \multirow[t]{3}{*}{ Metastasis-free survival, all diagnoses } & 1 & $98.5( \pm 2.0)$ & $95.5( \pm 3.5)$ & $95.5( \pm 3.5)$ \\
\hline & 2 & $85.3( \pm 4.5)$ & $83.0( \pm 4.9)$ & $76.3( \pm 5.9)$ \\
\hline & 3 & $64.6( \pm 5.7)$ & $58.1( \pm 5.9)$ & $54.9( \pm 6.1)$ \\
\hline \multirow[t]{3}{*}{ Metastasis-free survival, OSA } & 1 & $97.9( \pm 4.1)$ & $98.1 \%( \pm 3.7)$ & $98.1 \%( \pm 3.7)$ \\
\hline & 2 & $71.9( \pm 11.4)$ & $88.5( \pm 5.1)$ & $81.9( \pm 6.5)$ \\
\hline & 3 & $60.9( \pm 7.4)$ & $51.4( \pm 16.3)$ & $51.4( \pm 16.3)$ \\
\hline \multirow[t]{3}{*}{ Metastasis-free survival, CSA } & 1 & $100( \pm 0)$ & $87.3( \pm 11.6)$ & $87.3( \pm 11.6)$ \\
\hline & 2 & $91.3( \pm 4.5)$ & $81.1( \pm 16.7)$ & $67.8( \pm 22.1)$ \\
\hline & 3 & $57.1( \pm 15.9)$ & $67.5( \pm 10.6)$ & $62.7( \pm 11.8)$ \\
\hline \multirow[t]{3}{*}{ Metastasis-free survival, all other diagnoses } & 1 & $96.9( \pm 5.9)$ & $97.9( \pm 4.1)$ & $97.9( \pm 4.1)$ \\
\hline & 2 & $81.1( \pm 16.7)$ & $70.1( \pm 11.6)$ & $65.5( \pm 12.5)$ \\
\hline & 3 & $76.1( \pm 9.4)$ & $55.0( \pm 7.6)$ & $51.8( \pm 7.8)$ \\
\hline \multirow[t]{3}{*}{ Metastasis-free survival, Grade 2 OSA as high grade } & Low & $98.5( \pm 2.0)$ & $95.5( \pm 3.5)$ & $95.5( \pm 3.5)$ \\
\hline & Intermediate & $90.0( \pm 4.5)$ & $87.6( \pm 4.9)$ & $80.1( \pm 6.5)$ \\
\hline & High & $66.0( \pm 5.1)$ & $60.2( \pm 5.3)$ & $56.8( \pm 5.5)$ \\
\hline
\end{tabular}

$\mathrm{CI}=$ confidence interval $;$ OSA $=$ osteosarcoma CSA $=$ chondrosarcoma.

Fig. 1 Kaplan-Meier analysis of local recurrence for Grade 1,2 , and 3 bone sarcomas demonstrates no significant differences between groups (logrank $\mathrm{p}=0.864$ ).

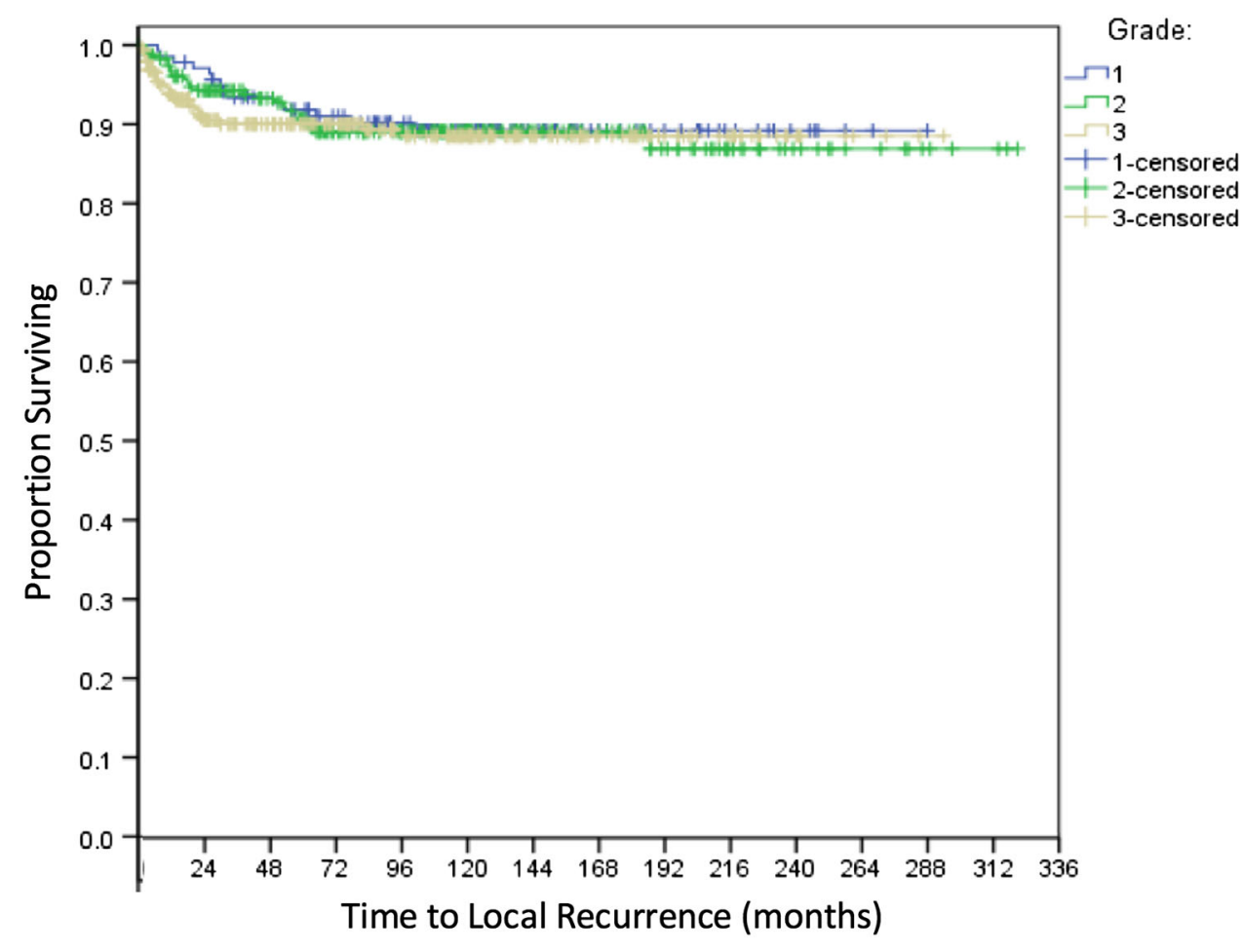


metastases. Axial tumor location was associated with higher rates of local recurrence compared with appendicular locations (hazard ratio [HR], 3.022; $\mathrm{p}<0.001 ; 95 \%$ confidence interval, 1.644-5.554); no other variables in the model were statistically significant.

Observed rates of metastasis for all diagnoses increased with sarcoma grade at 2, 5, and 10 years for the entire study population as well as for the subgroups of OSA, CSA, and other diagnoses (Table 2). The majority of metastases were pulmonary ( $\mathrm{n}=139$ [77\%]), whereas other less common sites included bone $(n=22[12 \%])$, soft tissue $(n=4[2 \%])$, lymph nodes $(\mathrm{n}=3[2 \%])$, brain $(\mathrm{n}=2[1 \%])$, and multiple areas $(n=10[6 \%])$. Only three Grade 1 sarcomas metastasized (one fibrosarcoma in GCT, one malignant GCT, and one malignant peripheral nerve sheath tumor of bone) with the latest event at 39 months (3 years). In both intermediate- and high-grade tumors, the rate of metastasis was greatest during the first 2 years, after which events continued at lower rates until 10 years; very late onset of pulmonary metastasis after 10 years occurred in only two cases, and both were associated with local recurrence. One patient was initially treated for a Grade 2 CSA that recurred locally at 187 months and metastasized at 191 months (16 years). The other patient underwent resection of a Grade 3 OSA that recurred locally at 19 months and metastasized at 215 months (18 years). Although tumor size was associated with metastasis ( $\mathrm{p}=0.029)$, the HR was quite small (HR, 1.041), indicating that it may not be clinically relevant; and no other variables in the Cox model were statistically significant. There was no detectable difference between the pattern and rate of metastasis to all sites compared with the subset of patients with metastasis only to the lungs. Because the majority of metastases are pulmonary and most of these are initially asymptomatic, our analysis focused only on lung metastasis to address the clinical issue of chest surveillance. Kaplan-Meier analysis with log-rank test confirmed worse metastasis-free survival in higher grade sarcomas ( $\mathrm{p}<0.001)$ (Fig. 2A). The proportion of patients who developed pulmonary metastasis was statistically different between Grade 2 and 3 CSA (logrank $\mathrm{p}<0.001$ ) but similar between Grade 2 and 3 OSA (log-rank $\mathrm{p}=0.010)$. In addition, for Grade 2 sarcomas, the proportion of patients with pulmonary progression was greater in those with OSA compared with CSA (log-rank $\mathrm{p}$ $=0.006)$, particularly in the first 2 years (Fig. $2 \mathrm{~B}-\mathrm{C})$. The numbers available for study were not sufficient to draw further definitive conclusions regarding other specific histologic diagnoses, although when grouped together, they followed similar patterns (Fig. 2D). These findings suggest that the metastatic behavior of Grade 2 OSA is more aggressive than other intermediate-grade sarcomas; therefore, we included Grade 2 OSA with other Grade 3 sarcomas as "high-grade" tumors for the remainder of the analysis, including calculation of survival rates for these adjusted groups (Table 2) and the development of our surveillance protocol (Fig. 3). The proportion of metastasis was significantly increased in high- compared with intermediate-grade sarcomas (log-rank $\mathrm{p}<0.001)$, suggesting that these groups should therefore be followed according to distinct schedules.

Comparing the incidence of new pulmonary metastasis per patient-year between grades generally supported current surveillance guidelines but did identify certain modifications that may increase the number of new pulmonary metastases detected per imaging examination performed (Table 3). The proportion of metastasis was substantially increased in high- compared with intermediate-grade sarcomas, suggesting that these groups should be followed at different intervals. Based on relative incidences of new pulmonary metastatic events per patient-year in these groups, we propose that chest imaging be performed as follows: annually only until 5 years for low-grade sarcomas; every 3 months for 2 years and annually from 2 to 10 years for intermediate-grade sarcomas; and every 3 months for 2 years, every 6 months from 2 to 5 years, and annually from 5 to 10 years for high-grade sarcomas (Table 4). This differs from current guidelines in that it increases the surveillance interval for intermediate-grade sarcomas between 2 and 5 years after treatment from 3 to 6 months, whereas the interval for high-grade sarcomas remains 3 months. To support this change, Fisher's exact test was performed to evaluate the difference between the incidences of new pulmonary metastases in intermediateversus high-grade sarcomas at 2 to 5 years after treatment, which was found to be significant ( $p=0.026)$.

\section{Discussion}

Because of the potential for development of local recurrence and distant metastasis after resection of a primary bone malignancy, ongoing surveillance is considered an essential aspect of sarcoma management. However, the potential benefits of early disease detection must be weighed against the missed work days, healthcare expenditures, and radiation exposure that increase with frequency of followup. Routine surveillance has been shown to improve survival in some malignancies [24] but not others $[7,8,15,16]$, and its role in sarcoma has not been firmly established [5, 13, 19].

Our study was limited by several factors, including the number of patients and low incidence of disease progression in our population. This combination resulted in an insufficient sample size to effectively compare rates of local recurrence between sarcoma diagnoses and rates of metastasis between diagnoses other than OSA and CSA. It 

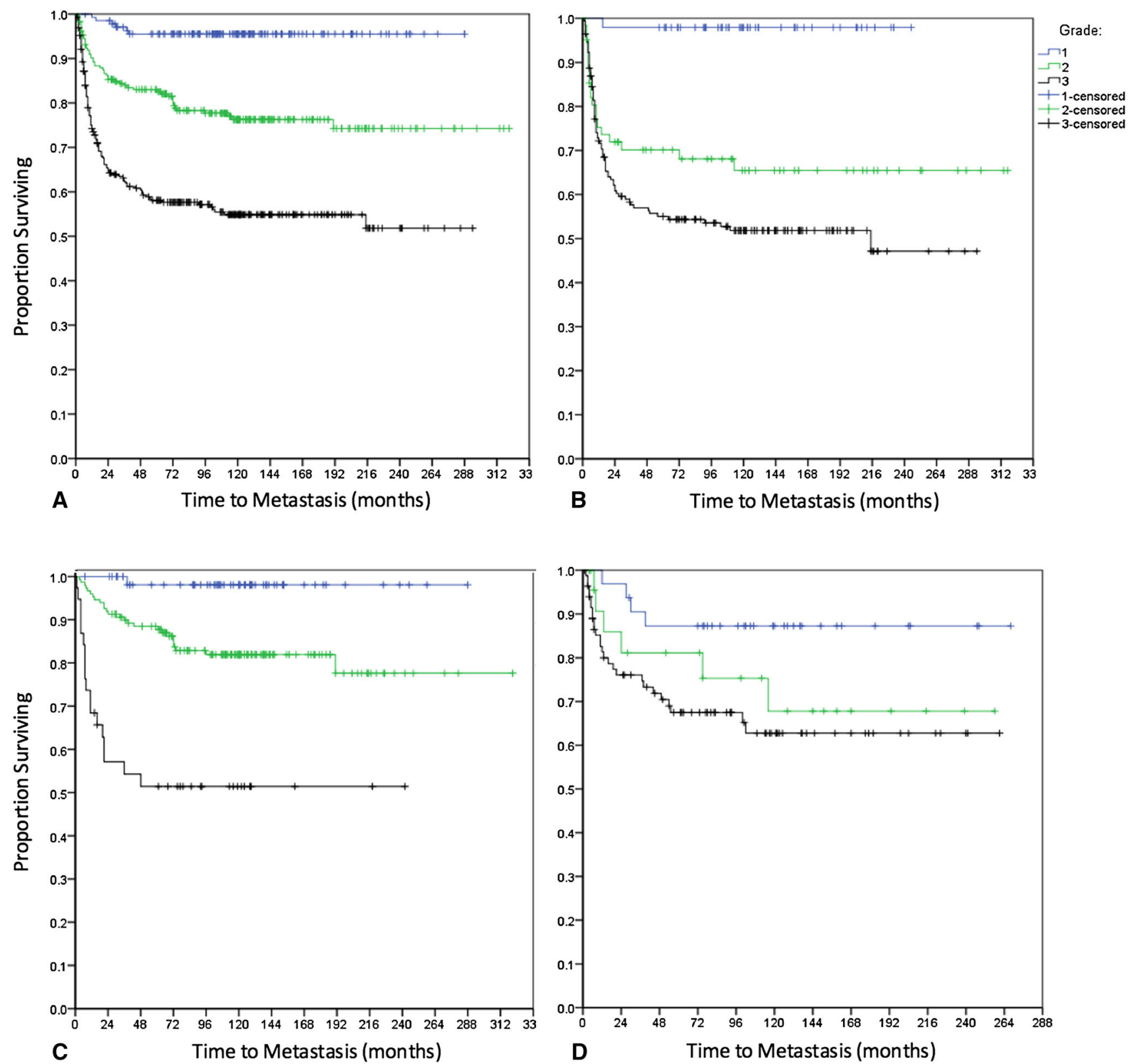

Fig. 2A-D Kaplan-Meier analysis of pulmonary metastasis by grade and diagnosis compares (A) all sarcomas by grade, (B) OSA by grade, (C) CSA by grade, and (D) all other sarcomas (non-OSA or CSA) by grade. Rates of metastasis were greater in higher grade sarcomas (log-

rank $\mathrm{p}<0.001$ ) and in Grade 2 OSA compared with Grade 2 CSA $(\log$-rank $\mathrm{p}=0.006)$

is also possible that delayed disease progression may occur beyond the period of observation; however, if our sample is representative, these very late events are so rare that ongoing surveillance is likely not indicated. Although we could not feasibly provide 10-year followup on all patients, the Kaplan-Meier analysis was designed to minimize the resulting bias by censoring patients, and patient-years of followup were used as the denominator for calculating event rates. Finally, our study was not designed to address the relative efficacy of imaging modalities (ie, chest

radiographs versus CT scans or local ultrasound versus MRI) nor the survival benefit of early detection, but to propose a surveillance schedule that would maximize the identification of new events per examination performed.

With the numbers of patients available for study, we could not detect a difference in local recurrence between the grades of sarcomas during the first 5 years despite the appearance of a slight increase in the first 2 years after treatment for the high-grade sarcomas. Local recurrence after 5 years was rare. Given that these events are often 
Fig. 3 Metastasis-free survival by sarcoma grade was adjusted by histologic tumor type such that Grade 2 OSA was combined with other Grade 3 sarcomas. Vertical reference lines at 2, 5, and 10 years and approximated lines of best fit for each sarcoma grade over each time interval are shown.

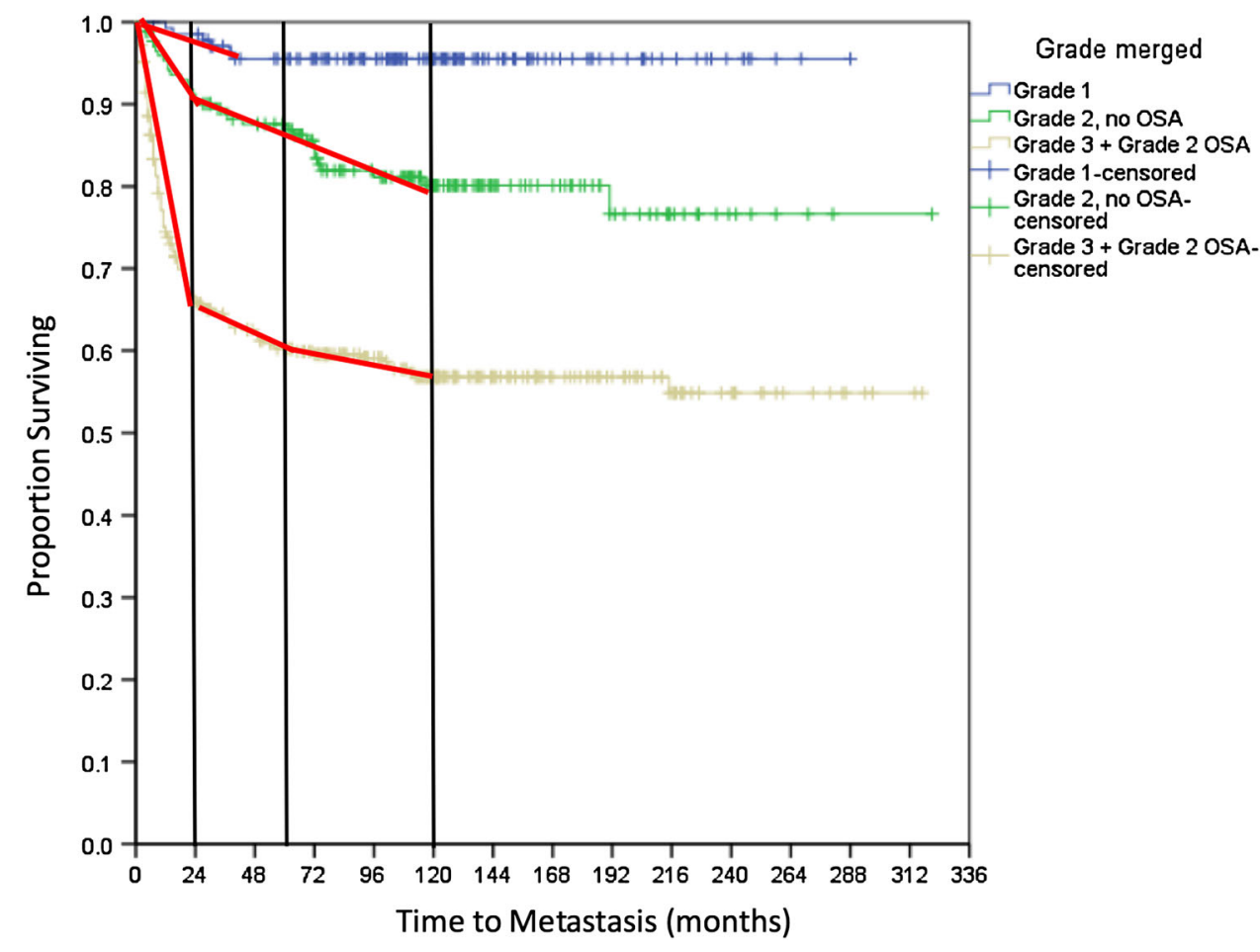

Table 3. Number and rates of new pulmonary metastatic events by grade across standard surveillance time intervals*

\begin{tabular}{|c|c|c|c|c|}
\hline Sarcoma grade & Years posttreatment & $\begin{array}{l}\text { New lung metastasis } \\
\text { events/patient-year }\end{array}$ & $\begin{array}{l}\text { Number of patients needed to screen to } \\
\text { detect } 1 \text { new metastatic event/year }\end{array}$ & Proposed screening frequency \\
\hline \multirow[t]{3}{*}{ Low } & $0-5$ & 0.023 & 44 & 12 months \\
\hline & $5-10$ & 0 & - & None \\
\hline & $>10$ & 0 & - & None \\
\hline \multirow[t]{4}{*}{ Int. } & $0-2$ & 0.52 & 2 & 3 months \\
\hline & $2-5$ & 0.029 & 35 & 12 months \\
\hline & $5-10$ & 0.026 & 39 & 12 months \\
\hline & $>10$ & 0.0038 & 264 & None \\
\hline \multirow[t]{4}{*}{ High } & $0-2$ & 0.66 & 2 & 3 months \\
\hline & $2-5$ & 0.11 & 10 & 6 months \\
\hline & $5-10$ & 0.018 & 56 & 12 months \\
\hline & $>10$ & 0.0012 & 834 & None \\
\hline
\end{tabular}

* The number of patients needed to be screened to detect one patient with new metastasis per year is listed.

detected on physical examination, performance of routine local imaging for the purpose of disease surveillance may not be beneficial, at least for most cases of extremity tumors. The limited data published on bone sarcomas suggest that local radiographs are not effective for surveillance [13, 23]. Previous studies also suggest that routine MRIs are not beneficial for soft tissue sarcomas with $89 \%$ to $97 \%$ of local recurrences detected by patients
$[26,27]$. Whether the effectiveness of self-monitoring is generalizable to bone sarcomas remains undetermined, but theoretically it may be even greater because these tumors are more frequently symptomatic. However, we also observed an increased local recurrence rate for axial tumors, which are more difficult to detect on physical examination. Thus, although all patients should be educated regarding the potential for local recurrence and 
Table 4. Proposed surveillance schedule for patients with bone sarcoma stratified according to rates of metastatic progression by grade and diagnosis

\begin{tabular}{lll}
\hline Grade & $0-2$ years & $2-5$ years \\
\hline Low grade (Grade 1) & 12 months & None \\
Intermediate grade (Grade 2 excluding OSA) & 3 months & 12 months \\
High grade (Grade 2 OSA and Grade 3) & 3 months & 6 months \\
\hline
\end{tabular}

OSA $=$ osteosarcoma.

instructed in self-examination, local imaging and/or more frequent followup should be considered after resection of axial tumors.

Pulmonary metastases are the most common manifestation of recurrent disease and tend to remain asymptomatic until reaching an advanced state. Prior work suggests that, if detected early, such disease may be more easily treatable and, in some cases, even curable with complete metastasectomy $[1,2]$. Some studies conclude that more aggressive pulmonary screening may facilitate earlier detection of lung metastases and improve survival for soft tissue sarcoma, particularly for higher risk patients $[5,6]$; others, however, either failed to confirm this advantage or demonstrated that less frequent scans are more appropriate for lower risk patients [13, 17, 20]. Although the optimal imaging frequency and modality to maximize survival remain unknown, it is generally agreed that patients with sarcoma require some form of surveillance that should be designed to maximize the number of recurrences detected per examination performed; therefore, understanding the frequency and timing of these events is necessary to determine optimal followup schedules.

Our findings suggest that pulmonary surveillance protocols could be adjusted according to the relative frequencies of new metastatic events based on sarcoma grade, diagnosis, and years since treatment. The incidence and timing of metastatic progression in the first 2 years were directly related to histologic grade, justifying more frequent chest imaging in higher grade sarcomas during that time period. However, the systemic recurrence pattern of Grade 2 OSA was more consistent with Grade 3 OSA than other Grade 2 diagnoses, suggesting that these patients would be most effectively followed according to highgrade protocols. Pulmonary metastasis of Grade 1 sarcomas was not observed after 39 months (3.25 years); thus, routine chest radiographs may be of limited use for these patients after 5 years of followup, and our protocol would have eliminated 10 imaging studies per patient $(42 \%$ reduction). By contrast, metastasis in intermediate- and high-grade sarcomas decreased after 2 years but continued until 10 years with only two events thereafter, both associated with local recurrence. We did note a difference in the incidence of new pulmonary metastasis between these two groups, and our proposed protocol, which distinguishes between the two, would have decreased the number of chest studies by six per patient over 10 years $(32 \%$ reduction compared with our previous protocol). Although performing a survival or cost-benefit analysis was beyond the scope of this study, the theoretical advantages of reducing unnecessary testing include decreased healthcare expenditures, radiation exposure, number of office visits, and time away from work for sarcoma survivors. We observed only two cases of very late disease progression beyond 10 years, both associated with local recurrence, illustrating the value of continued long-term clinical followup. Any new patient symptoms deserve investigation and serious consideration and, if local recurrence is detected, restaging should be performed and surveillance reinitiated.

Although our study may have limitations, it offers the only published data on metastatic patterns in patients with bone sarcoma and the only surveillance protocol based on evidence beyond expert opinion. ESMO states that "strict rules cannot be provided in the absence of any formal validation" and the committee therefore only provides suggestions for followup with no referenced literature [8]. Similarly, the NCCN guidelines for surveillance of soft tissue sarcomas offer no supporting evidence, stating instead that "very limited data is available in the literature on effective surveillance strategies" [25]. Finally, the NCCN guidelines for bone cancer cite a single publication on a cohort of patients with CSA, the primary objective of which was to identify prognostic factors for survival rather than report patterns of metastases [14]. Thus, our study, although imperfect, offers stronger data, which allowed development of an evidence-based surveillance protocol than currently exists for sarcoma.

In conclusion, it is apparent that more studies with larger patient numbers are needed to develop an evidence-based followup protocol for patients with bone sarcoma. We propose a guideline that differs from the ESMO and NCCN guidelines and will reduce the number of chest images for a given patient over a 10-year period. We do not suggest that this is the optimal protocol nor that it will detect local or 
distant metastases sooner or more accurately than other regimens. We hope that a data-driven approach for followup will encourage other groups to use or modify this protocol, eventually developing a more rational guideline that enhances early detection of recurrence, reduces radiation exposure, and lowers cost of surveillance.

\section{References}

1. Al-Hussaini H, Hogg D, Blackstein ME, O'Sullivan B, Catton CN, Chung PW, Griffin AM, Hodgson D, Hopyan S, Kandel R, Ferguson PC, Wunder JS, Gupta AA. Clinical features, treatment, and outcome in 102 adult and pediatric patients with localized high-grade synovial sarcoma. Sarcoma. 2011;2011:231789.

2. Aljubran AH, Griffin A, Pintilie M, Blackstein M. Osteosarcoma in adolescents and adults: survival analysis with and without lung metastases. Ann Oncol. 2009;20:1136-1141.

3. Beitler AL, Virgo KS, Johnson FE, Gibbs JF, Kraybill WG. Current follow-up strategies after potentially curative resection of extremity sarcomas: results of a survey of the members of the Society of Surgical Oncology. Cancer. 2000;88:777-785.

4. Berrington de Gonzalez A, Mahesh M, Kim KP, Bhargavan M, Lewis R, Mettler F, Land C. Projected cancer risks from computed tomographic scans performed in the United States in 2007. Arch Intern Med. 2009;169:2071-2077.

5. Cho HS, Park IH, Jeong WJ, Han I, Kim HS. Prognostic value of computed tomography for monitoring pulmonary metastases in soft tissue sarcoma patients after surgical management: a retrospective cohort study. Ann Surg Oncol. 2011;18:3392-3398.

6. Chou YS, Liu CY, Chen WM, Chen TH, Chen PC, Wu HT, Chiou HJ, Shiau CY, Wu YC, Liu CL, Chao TC, Tzeng CH, Yen CC. Follow-up after primary treatment of soft tissue sarcoma of extremities: impact of frequency of follow-up imaging on disease-specific survival. J Surg Oncol. 2012;106:155-161.

7. Emens LA, Davidson NE. The follow-up of breast cancer. Semin Oncol. 2003;30:338-348.

8. ESMO/European Sarcoma Network Working Group. Bone sarcomas: ESMO Clinical Practice Guidelines for diagnosis, treatment and follow-up. Ann Oncol. 2014;3:113-123.

9. Gerrand CH, Billingham LJ, Woll PJ, Grimer RJ. Follow up after primary treatment of soft tissue sarcoma: a survey of current practice in the United Kingdom. Sarcoma. 2007;2007:34128.

10. Goda JS, Ferguson PC, O'Sullivan B, Catton CN, Griffin AM, Wunder JS, Bell RS, Kandel RA, Chung PW. High-risk extracranial chondrosarcoma: long-term results of surgery and radiation therapy. Cancer. 2011;117:2513-2519.

11. Goel A, Christy ME, Virgo KS, Kraybill WG, Johnson FE. Costs of follow-up after potentially curative treatment for extremity soft-tissue sarcoma. Int J Oncol. 2004;25:429-435.

12. Grimer R, Athanasou N, Gerrand C, Judson I, Lewis I, Morland B, Peake D, Seddon B, Whelan J. UK guidelines for the management of bone sarcomas. Sarcoma. 2010;2010:317462.
13. Korholz D, Verheyen J, Kemperdick HF, Gobel U. Evaluation of follow-up investigations in osteosarcoma patients: suggestions for an effective follow-up program. Med Pediatr Oncol. 1998;30:52-58.

14. Lee FY, Mankin HJ, Foudren G, Gebhardt MC, Springfield DS, Rosenberg AE, Jeenings LC. Chondrosarcoma of bone: an assessment of outcome. J Bone Joint Surg Am. 1999;3:326-338.

15. Meyerhardt JA, Mayer RJ. Follow-up strategies after curative resection of colorectal cancer. Semin Oncol. 2003;30:349-360.

16. Meyers MO, Yeh JJ, Frank J, Long P, Deal AM, Amos KD, Ollila DW. Method of detection of initial recurrence of stage II/III cutaneous melanoma: analysis of the utility of follow-up staging. Ann Surg Oncol. 2009;16:941-947.

17. Miller BJ, Carmody Soni EE, Reith JD, Gibbs CP, Scarborough MT. CT scans for pulmonary surveillance may be overused in lower-grade sarcoma. Iowa Orthop J. 2012;32:28-34.

18. NCCN Guidelines. Cancer. Available at: http://download.bioon. com.cn/view/upload/201302/27103213_7950.pdf. Accessed Date 2 Apr 2016.

19. Postovsky S, Barzilai M, Meller I, Kollander Y, Futerman B, Ben Arush MW. Does regular follow-up influence the survival of patients with sarcoma after recurrence? The Miri Shitrit pediatric oncology department experience. J Pediatr Hematol Oncol. 2008;30:189-195.

20. Puri A, Gulia A, Hawaldar R, Ranganathan P, Badwe RA. Does intensity of surveillance affect survival after surgery for sarcomas? Results of a randamized noninferiority trial. Clin Orthop Relat Res. 2014;472:1568-1575

21. Sawamura C, Matsumoto S, Shimoji T, Okawa A, Ae K. How long should we follow patients with soft tissue sarcomas? Clin Orthop Relat Res. 2014;472:842-848.

22. Smith TJ, Hillner BE. Bending the cost curve in cancer care. $N$ Engl J Med. 2011;364:2060-2065.

23. Takeuchi A, Lewis VO, Satcher RL, Moon BS, Lin PP. What are the factors that affect survival and relapse after local recurrence of osteosarcoma? Clin Orthop Relat Res. 2014;472:3188-3195.

24. Tanner EJ, Chi DS, Eisenhauer EL, Diaz-Montes TP, Santillan A, Bristow RE. Surveillance for the detection of recurrent ovarian cancer: survival impact or lead-time bias? Gynecol Oncol. 2010;117:336-340.

25. von Mehren M, Benjamin RS, Bui MM, Casper ES, Conread EU 3rd, Delaney TF, Ganjoo KN, George S, Gonzalez, Heslin MJ, Kane JM 3rd, Mayerson J, McGarry SV, Meyer C, O’Donnell RJ, Paz B, Pfeifer JD, Pollock RE, Randell RL, Riedel RF, Schuetze S, Schupak KD, Schwartz HS, Shanker S, Van Tine BA, Wayne J, Sunder H, McMillian NR. Soft tissue sarcoma, version 2.2012: featured updates to the NCCN guidelines. J Natl Compr Canc Netw. 2012;2:951-960.

26. Whooley BP, Gibbs JF, Mooney MM, McGrath BE, Kraybill WG. Primary extremity sarcoma: what is the appropriate followup? Ann Surg Oncol. 2000;7:9-14.

27. Whooley BP, Mooney MM, Gibbs JF, Kraybill WG. Effective follow-up strategies in soft tissue sarcoma. Semin Surg Oncol. 1999;17:83-87. 